\title{
Targeting the BDNF/TrkB pathway for the treatment of tumors (Review)
}

\author{
LINGBIN MENG $^{1}$, BAOQIONG LIU ${ }^{1}$, RUI JI ${ }^{2}$, XIN JIANG $^{3}$, XUEBO YAN $^{4}$ and YING XIN ${ }^{5}$ \\ ${ }^{1}$ Department of Internal Medicine, Florida Hospital, Orlando, FL 32803; ${ }^{2}$ Department of Biology, Valencia College, Orlando, \\ FL 32825, USA; ${ }^{3}$ Department of Radiation Oncology, The First Hospital of Jilin University, Changchun, Jilin 130021; \\ ${ }^{4}$ Department of Respiratory Medicine, The First Affiliated Hospital of Anhui Medical University, Hefei, Anhui 230000; \\ ${ }^{5}$ Key Laboratory of Pathobiology, Ministry of Education, Jilin University, Changchun, Jilin 130021, P.R. China
}

Received June 28, 2018; Accepted December 12, 2018

DOI: $10.3892 / \mathrm{ol} .2018 .9854$

\begin{abstract}
Neurotrophins are a family of growth factors that regulate neural survival, development, function and plasticity in the central and the peripheral nervous system. There are four neurotrophins: nerve growth factor (NGF), brain-derived neurotrophic factor (BDNF), neurotrophin-3 (NT-3) and NT-4. Among them, BDNF is the most studied due to its high expression in the brain. Over the past two decades, BDNF and its receptor tropomyosin receptor kinase $\mathrm{B}$ (TrkB) have been reported to be upregulated in a wide range of tumors. This activated signal stimulates a series of downstream pathways, including phosphoinositide 3-kinase/protein kinase B, Ras-Raf-mitogen activated protein kinase kinase-extracellular signal-regulated kinases, the phospholipase-C- $\gamma$ pathway and the transactivation of epidermal growth factor receptor. Activation of these signaling pathways induces oncogenic effects by increasing cancer cell growth, proliferation, survival, migration and epithelial to mesenchymal transition, and decreasing anoikis, relapse and chemotherapeutic sensitivity. The present review summarizes recent findings to discuss the role of BDNF in tumors, the underlying molecular mechanism, targeting Trk receptors for treatment of cancers and its potential risk.
\end{abstract}

\section{Contents}

1. Introduction

2. BDNF and TrkB receptor

3. Oncogenic role of BDNF in tumor

Correspondence to: Dr Ying Xin, Key Laboratory of Pathobiology, Ministry of Education, Jilin University, 126 Xinmin Street, Changchun, Jilin 130021, P.R. China

E-mail: rji@valenciacollege.edu

Key words: brain-derived neurotrophic factor, tropomyosin receptor kinase B, survival, apoptosis, anoikis, chemotherapy resistance, phosphoinositide 3-kinase, phospholipase-C- $\gamma$, mitogen-activated protein kinase
4. Molecular pathways of BDNF/TrkB's oncogenic role

5. Clinical trials of targeting Trk receptors for treatment of cancer

6. Potential side effects of targeting BDNF/TrkB pathway to treat cancer

7. Conclusion and discussion

\section{Introduction}

Neurotrophins are a family of proteins that regulate neuron differentiation, survival, dendritic pruning, patterning of innervation, synaptic function and plasticity in the central and the peripheral nervous system $(1,2)$. There are four neurotrophins: nerve growth factor (NGF), brain-derived neurotrophic factor (BDNF), neurotrophin-3 (NT-3) and NT-4. They have two types of receptors: the p75 neurotrophin receptor and tropomyosin receptor kinases (Trk) (2). P75 is the receptor for all four neurotrophins. Regarding Trk receptors, NGF binds TrkA; BDNF and NT-4 bind TrkB; and NT-3 mainly binds TrkC (2) (Fig. 1).

Initially, neurotrophins and their receptors were thought to be expressed only in nervous system but further studies showed they are also expressed by macrophages, endocrine cells, immune cells, smooth and striated muscle fibers $(3,4)$. Recently, neurotrophins and their Trk receptors, especially BDNF and TrkB, were found to be highly up-regulated and play a vital role in various cancers, including breast, lung, colon-rectum, pancreas, prostate, liver, myelomas and lymphoid tumors (5). Activation of these Trk receptors elicits a series of downstream signalings, including PI3K/Akt, Ras-Raf-MEK-ERK, PLC $\gamma$ pathway, transactivation of EGFR, etc. As a result, these pathways exhibit oncogenic effects by promoting cancer cell's growth, proliferation, survival, migration, epithelial to mesenchymal trasition, anoikis, relapse and chemotherapeutic sensitivity (6-12). Drugs targeting these Trk receptors have been put into clinical trials for cancer therapy and promising results have been achieved with moderate side effects (13).

This review will summarize all recent findings about the role of BDNF/TrkB in tumor and its underlying downstream pathways. We will also conclude and discuss clinical trials of targeting Trk receptors for treatment of cancers and the potential risk. 


\section{BDNF and TrkB receptor}

Among the 4 neurotrophins, BDNF is the most abundant growth factor in the brain, which plays an important role in sustaining physiological processes of the brain. For example, BDNF regulates dendritic branching and dendritic spine morphology $(14,15)$, as well as synaptic plasticity and long-term potentiation (LTP) (16). BDNF also modulates hypothalamic metabolic function, further reflecting the diversity of its role in the brain $(17,18)$.

It has been revealed that BDNF is important in the developmental and mature taste system, by supporting survival of taste cells and geniculate ganglion neurons, and maintaining and guiding taste nerve innervations (19-21). These results demonstrated BDNF exhibits crucial effects in both of the central and peripheral nervous system. Another study also showed that BDNF/TrkB pathway may be involved in maintaining adult hippocampal neurogenesis by promoting survival, proliferation, and neural differentiation of neural stem cells $(22,23)$. This function and underlying mechanism is comparable to BDNF's role in cancer (23-25).

Recently, lots of evidences showed BDNF and its receptor TrkB play a vital role in tumor pathology (26-28). TrkB is a type of receptor tyrosine kinases (RTKs) and some RTKs were characterized as oncogenes (29). Preclinical trials of target therapies on these RTKs showed promising results (13). Recent reports indicate that BDNF/TrkB pathway has an important function in neural tumors, such as neuroblastoma (30). Further studies have shown that BDNF/TrkB is oncogenic not only in neurogenic original tumors (31), but also in other tumors outside of the neural system $(32,33)$.

In addition to TrkB, p75 is a receptor for a precursor form of BDNF (pro-BDNF), which can be cleaved to form the mature form by metalloproteinases (34). Unlike TrkB, which is the receptor for the mature form of BDNF (35), the role of p75 is not well established in tumors. It is found to be overexpressed in glioblastoma $(34,36)$, melanoma $(37,38)$ and breast cancer (6), implying it may exhibit an oncogenic role. However, it also displays an oncolytic role by suppressing tumor cell proliferation and migration in bladder (39), hepatocellular (40) and gastric cancers (41). Considering p75's controversial role in tumor, we will only summarize the oncogenic role of BDNF/TrkB pathway for the following sections.

\section{Oncogenic role of $\mathrm{BDNF}$ in tumor}

As said above, BDNF's oncogenic role in cancer has initially been characterized in neuroblastoma, a type of cancer in nervous tissue (30). It has ever been demonstrated Tyro3, Axl and Mertk (TAM) receptor tyrosine kinases promote neurogenesis by supporting neural stem cell survival, proliferation and neuronal differentiation $(22,42)$. Removal of TAM receptors leads to a significantly-reduced level of neurogenesis and BDNF expression, indicating TAM receptors support neurogenesis by activating BDNF pathway (23). Some scientists also concluded BDNF mediates development, migration, differentiation and survival of newborn neurons (43). Further on, high levels of BDNF were found in neuroblastoma cells and were discovered to be linked with better prognosis of neuroblastoma (44). An increase of BDNF and TrkB signaling in neuroblastoma cells may represent an autocrine system to support tumor growth, invasion and metastasis. Mover, BDNF/TrkB pathway was implied to induce angiogenesis in neuroblastoma. It was found that BDNF could stimulate neovascularization through recruitment of TrkB-expressing endothelial progenitor cells $(45,46)$. Lastly, this signaling was also demonstrated to promote resistance to chemotherapy in neuroblastoma cells (47).

As evidence is accumulated, BDNF/TrkB signaling is universally considered to have oncogenic consequences. It has been found BDNF/TrkB are up-regulated in countless types of cancers, such as breast cancer, carcinoid, cervical, colorectal, glioma, liver, lung (6-12). Some studies even revealed BDNF might be an important prognostic factor for cancers $(12,48-50)$. Recently, BDNF/TrkB pathway has been demonstrated to transactivate EGFR, a growth factor receptor commonly up-regulated in many cancers $(43,51)$. This transactivation is important for proliferation and migration of embryonic cortical neurons, lung cancer cells and ovarian cancer cells $(43,51,52)$. Administration of BDNF prevents the oncolytic role of EFGR inhibition in colon cancer. Besides, BDNF and EGFR seems to compensate for each other so that dual inhibition of the two pathways works effectively to suppress colon cancer cell proliferation (53).

BDNF/TrkB can also decrease a cancer cell's sensitivity to chemotherapy. It has been reported that BDNF increases the survival of neuroblastoma cells from cisplatin, etoposide and vinblastine in a dose-dependent manner $(54,55)$. Treatment with antibodies against BDNF made mice more susceptible to chemotherapy in models of breast cancer (6), uterine sarcoma (56), and neuroblastoma $(57,58)$. BDNF administration was demonstrated to cause chemotherapeutic resistance in head and neck squamous cell carcinoma (59). This protective role of BDNF from chemotherapy was possibly due to its ability to support proliferation and survival of cancer cells (55).

In addition, BDNF/TrkB pathway promotes resilience against the programmed death of anchorage dependent cancer cells. This programmed death of cancer cells, known as anoikis, is important to fight against many types of cancers. As solid tumors metastasize from the original sites and migrate to other regions with plentiful nutrients, tumor cells may undergo anoikis (60). However, some tumor cells can have a mesenchymal transition from an epithelial nature so that they survive short travel through the blood to distant organs (61). Interestingly, BDNF/TrkB has been reported to regulate the resistance to anoikis of several cancers since up-regulation of BDNF/TrkB was found in metastatic tumor cells. Nevertheless, no activation of BDNF/TrkB pathway was observed in non-metastatic tumor cells or tumor cells that fail to survive through metastasis $(60,62,63)$. This supports how $\mathrm{BDNF} / \mathrm{TrkB}$ signaling may perhaps play a crucial role in the progression and invasion of malignant tumors.

Finally, BDNF/TrkB pathway has been implied to mediate cancer reformation after successful treatment of cancer. Scientists overexpressed TrkB in a neural crest-derived cell line and implanted them into mice. These cells formed tumors 10 days after implantation and killed all mice within one week after tumor formation (31). Cancer stem cells are similar to neural crest-derived cells. They divide slowly but can 


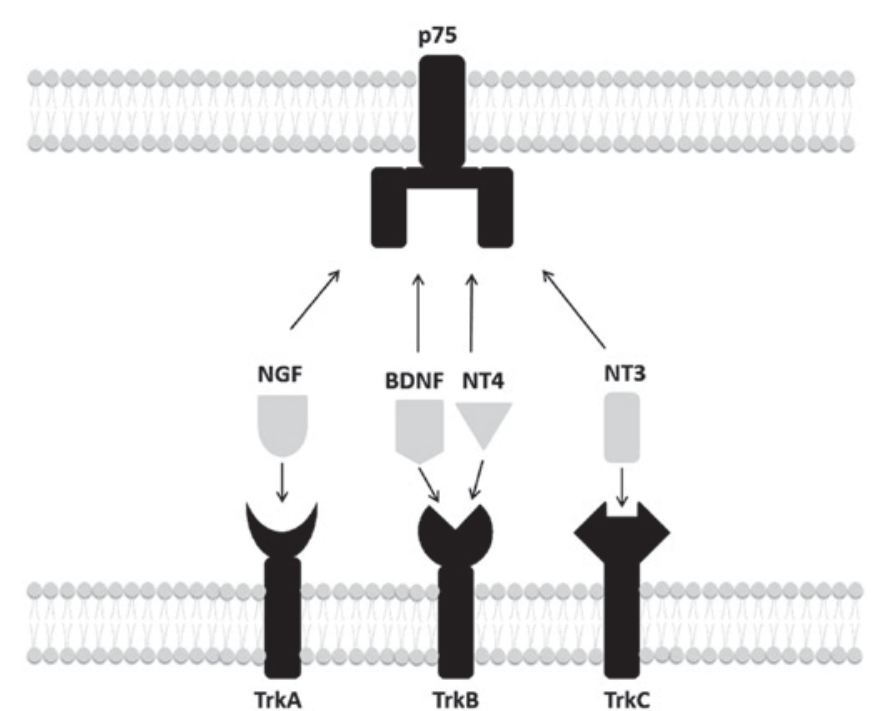

Figure 1. Neurotrophins and their receptors. NGF binds to TrkA receptors, BDNF and NT4 bind to TrkB, and NT3 mainly binds to TrkC receptors. All of these neurotrophins also bind to the low-affinity receptor p75. NGF, nerve growth factor; BDNF, brain-derived neurotrophic factor; NT, neurotrophin; Trk, tropomyosin receptor kinase.

turn into cancer cells under some condition. Chemotherapy can kill rapidly-dividing tumor cells but can't target these slowly-dividing cells. It has been shown that TrkB-positive cancer stem cells can cause tumor reformation after successful treatment of mice with triple-negative breast cancers (32). These findings demonstrate the importance of continuous treatment with TrkB inhibitor after successful removal of tumor cells with chemotherapy.

\section{Molecular pathways of $\mathrm{BDNF} / \mathrm{TrkB}$ 's oncogenic role}

As discussed above, it has been observed that BDNF and TrkB levels increase in many types of cancers, conferring aggressive phenotypes due to their resistance to chemotherapeutic agents (64). BDNF binds its receptor TrkB and triggers a cascade of signals, including PI3K/Akt, Ras-Raf-MEK-ERK, PLC $\gamma$ pathway, transactivation of EGFR, Jak/STAT, nuclear factor kappa-light-chain-enhancer of activated B cells (NF-kB), Urokinase-type plasminogen activator (UPAR)/UPA, Wnt/ $\beta$-catenin and Vascular endothelial growth factor (VEGF) pathways, etc. Among these pathways, the first 4 are mostly studied and therefore will be discussed in this review and summarized as shown in Fig. 2.

PI3K/Akt can be activated by BDNF/TrkB and then leads to production of pro-migratory, anti-apoptotic and pro-survival proteins $(65,66)$. Some recent studies have indicated that TrkB receptor activation induces phosphorylation of tyrosine 705 of STAT3, which then activates PI3K/Akt (67). This pathway will activate the mammalian target of rapamycin complex 1 (mTORC1), resulting in increased protein synthesis and cell survival by direct phosphorylation of its effectors, such as the ribosomal S6 kinasel (S6K1), and elF4E-binding proteins (4E-BPs) to terminate binding to elF4E and relieve the block on translation $(68,69)$. Besides, PI3K/Akt pathway can transduce to amplify hypoxia-inducible factor 1-alpha (H1F1a), which is a transcriptional activator of TrkB expression. This

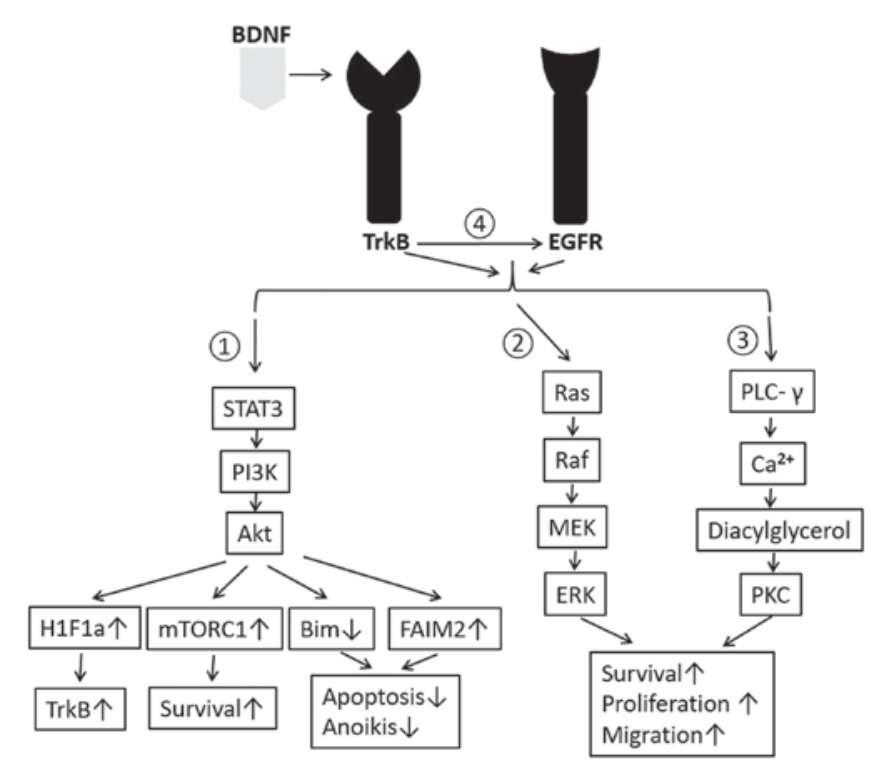

Figure 2. Summarized molecular pathway of BDNF/TrkB's oncogenic role. Four signaling pathways are discussed in the present review: i) Phosphorylation of STAT3 activates the PI3K/Akt signaling pathway, which will then amplify H1F1a to positively control TrkB expression, stimulate mTORC1 for the expression of pro-survival proteins and reduce Bim, but increase FAIM2 in order to inhibit apoptosis or anoikis; ii) Ras-Raf-MEK-ERK signaling is activated in order to promote the survival, proliferation and migration of cancer cells; iii) activated expression of PLC- $\gamma$ causes the release of calcium ions from intracellular compartments and the generation of diacylglycerol. Diacylglycerol activates PKC, which exerts oncogenic effects; and iv) Transactivation of EGFR by BDNF/TrkB can also regulate cancer by activating the above 3 signaling pathways. An upwards pointing arrow after each signal protein indicates upregulation of expression level while a downwards facing arrow represents decreased expression. BDNF, brain-derived neurotrophic factor; Trk, tropomyosin receptor kinase; STAT3, signal transducer and activator of transcription 3; PI3K, phosphoinositide 3-kinase; PLC- $\gamma$, phospholipase-C- $\gamma$, mitogen-activated protein kinase. PKC, protein kinase C; H1F1a, hypoxia-inducible factor $1 \alpha$; FAIM2, Fas apoptotic inhibitory molecule 2; MEK, mitogen activated protein kinase kinase; ERK, extracellular signal-regulated kinases.

positive feedback loop aggravates and extends BDNF/TrkB's effect on tumor $(67,70)$.

As a universal attenuator of chemotherapeutic efficacy, BDNF/TrkB exhibits this role by mediating PI3K/Akt pathway, since inhibition of PI3K abrogated BDNF's ability to protect cancer cells from etoposide (55). PI3K/Akt pathway promotes resistance to extrinsic apoptosis through down-regulation of Bim, a pro-apoptotic protein that facilitates mitochondrial-mediated or intrinsic apoptosis $(59,71)$. In addition, PI3K/Akt pathway is involved in up-regulation of Fas apoptotic inhibitory molecule 2 (FAIM2), which works to inhibit Fas-mediated Caspase-8-dependent apoptosis (72). The two possible pathways also explain how BDNF promotes resistance to anoikis through activation of PI3K/Akt.

BDNF/TrkB pathway has also been shown to transactivate EGFR even without the endogenous EGF ligand (43) (Fig. 2). In vitro study demonstrated administering BDNF leads to expected TrkB phosphorylation and also EGFR phosphorylation no matter if EGF is present in the culture media or not (43). Transactivation of EGFR stimulates expression of PLC- $\gamma$, which then causes the release of calcium ions from intracellular compartments and the generation of diacylglycerol. Diacylglycerol can activate protein kinase C (PKC), 
which is linked to carcinogenesis and maintenance of malignant phenotype (73). Besides, EGFR can result in the progression of cancer cells through G1 phase and into $\mathrm{S}$ phase by regulating the cyclin dependent kinases (CDK) and the cyclins (74). EGFR can also cause Ras activation, which involves large number of protein factors, including Raf, mitogen-activated protein kinase (MAPK), cytosolic kinases and nuclear transcription factors $(75,76)$. Activation of Ras will in turn accelerate cell-cycle progression and contribute to poor prognosis of patients with cancers $(12,77)$. Moreover, it is possible that transactivation of EGFR regulates cancer through PI3K/Akt pathway, as shown in breast cancer (78), head and neck cancer (79), and prostate cancer (80).

Finally, BDNF/TrkB is considered to be able to directly activate Ras and PLC- $\gamma$ pathways, both of which play a vital role in a wide range of cancers (Fig. 2). In the central nervous system, it is well documented that BDNF/TrkB activates the Ras-Raf-MEK-ERK signaling and regulates the neuronal differentiation (81). BDNF/TrkB pathway is also demonstrated to regulate synaptic plasticity by promoting the PLC $\gamma$-mediated expression of protein kinase C (82). Recent studies showed Ras and PLC $-\gamma$ mediated oncogenic role may be triggered by BDNF/TrkB. For example, BDNF/TrkB activates NF- $\kappa$ B expression through stimulation of PLC $\gamma$ and therefore enhances ovarian cancer cell survival by suppressing anoikis (83). $\mathrm{BDNF} / \mathrm{TrkB}$ is also demonstrated to promote epithelia-mesenchymal transition, as well as the migration and invasion of cervical cancer by activating Ras-Raf-MEK-ERK pathway (84).

\section{Clinical trials of targeting Trk receptors for treatment of cancer}

Scientists have developed two highly potent and selective TrkB inhibitors, cyclotraxin-B (85) and antinuclear antibodies (ANA)-12 (86), which can inhibit TrkB and its downstream processes. However, they are not applied to clinical trials since targeting all Trk receptors seems more promising to treat cancer. Like TrkB, TrkA and TrkC are up-regulated in many types of cancers and demonstrated to be oncogenic as well (13). For example, Light et al (87) reported TrkA up-regulation in neuroblastomas was associated with poor prognosis, while activated expression and signaling of TrkC corresponded to a more aggressive and invasive neuroblastoma. Besides, the kinase domain of the three receptors are remarkably conservative. TrkB and TrkC share $100 \%$ identical residues in the ATP binding sites, and there is only a 2-residue difference between TrkA and TrkB (88). Considering kinase domain determines their activity, it is reasonable and applicable to design inhibitors targeting the conservative kinase domain. Based on the fact that the three Trk receptors share similar kinase domain and all have oncogenic role, drugs targeting all of them were designed and applied to clinical trials.

Entrectinib is an ATP-competitive inhibitor of the Trk proteins, c-ros oncogene 1 (ROS1), and anaplastic lymphoma kinase (ALK). It is currently being investigated in multiple phase II studies, including breast cancer, renal cancer, ovarian cancer, non-small cell lung cancer (NSCLC), and sarcomas (89). Promising results have been reported, including increased objective response rate, median progression-free survival rate and overall survival rate. Interestingly, Entrectinib has also shown the efficacy to treat brain tumors, implying that it can penetrate blood-brain barrier (BBB) (90). Similar to Entrectinib, Larotrectinib is another pan-Trk inhibitor which is able to penetrate $\mathrm{BBB}$ and shows positive results in multiple phase II clinical trials, including glioblastoma, small cell lung cancer (SCLC), colorectal cancers, melanoma pancreatic and ovarian $(91,92)$. A table was made to summarize all clinical trials for the two Trk inhibitors (Table I).

Cabozantinib is an orally bioavailable small molecule inhibitor of Trk receptors, c-Met, RET, ROS1, ALK, and vascular endothelial growth factor 2 (VEGFR2) with approved treatment for metastatic medullary thyroid cancer and prostate cancer (13). Recently, Cabozantinib was approved as an anti-angiogenic therapy for advanced renal cell carcinoma, by eliciting significant improvements in response rates, progression-free survival, and overall survival $(93,94)$. Currently, more clinical trials are underway to evaluate its role in CNS tumors, like gliomas (95). Considering its capacity of penetrating BBB, promising results are expected.

\section{Potential side effects of targeting BDNF/TrkB pathway to treat cancer}

Though the clinical trials received promising results, we should not ignore the potential side effects of targeting BDNF/TrkB for cancer treatment. As a neurotrophin factor in the nervous system, BDNF regulates multiple processes including neuron differentiation, survival, dendritic pruning, patterning of innervation, synaptic function and plasticity $(1,2)$. Our lab has demonstrated BDNF plays a vital role in the central and peripheral nervous system by regulating the developmental and mature taste system (19-21), and maintaining adult hippocampal neurogenesis $(22,23)$. A recent clinical trial even showed elevation of BDNF levels by using CX1846 can correct age-related issues (96). Therefore, we should be extremely careful when targeting BDNF/TrkB to treat cancer. Doses of BDNF/TrkB inhibitors and side effects of nervous system should be closely monitored. Dysfunction of central nervous system may be expected, like memory loss, ataxia, anhedonia, lethargy and depression (97). As a result, it is necessary to specifically target tumors with administration of BDNF/TrkB inhibitors. Gene delivery using viral vectors may be a good option since it can specifically target tumor cells with appropriate promoters.

Besides, recent studies suggest that BDNF overexpression in the hypothalamus may have an oncolytic effect. It is found that mice with enriched environmental (EE) housing had high expression of BDNF in the hypothalamus and also got augmented T-cell cytotoxicity. This increased anti-tumor immune response was abrogated by hypothalamic knockdown of BDNF, implying BDNF mediates the oncolytic effects of EE housing (98). Besides, tumors of EE mice had reduced expression of several pro-survival proteins like VEGF, IGF-1 and p-ERK, which normally confer resistance to chemotherapeutic agents. These results were derived from mice transplanted with breast cancer cells (99), melanoma cancer cells (100), and even glioma cells (101). It is suggested that up-regulation of BDNF in the central nervous system may have an effect of oncolysis rather than oncogenesis, even if the tumor is within the central nervous system. The underlying mechanism may be due to that BDNF supports survival and 
Table I. Ongoing clinical trials for Entrectinib and Larotrectinib in different types of cancers.

\begin{tabular}{llll}
\hline NCT identifier Drug Phase Cancer type Status \\
\hline
\end{tabular}

NCT02568267 Entrectinib II Breast cancer, cholangiocarcinoma, colorectal cancer, head and neck Recruiting
neoplasms, lymphoma, large-cell, anaplastic, melanoma, neuroendocrine tumors, non-small cell lung cancer, ovarian cancer, pancreatic cancer, papillary thyroid cancer, primary brain tumors, renal cell carcinoma, sarcomas, salivary gland cancers, and adult solid tumor

$\begin{array}{llll}\text { NCT03330990 } & \text { Entrectinib } & \text { I } & \text { Advanced solid tumor } \\ \text { NCT02650401 } & \text { Entrectinib } & \text { I } & \text { Solid tumors, CNS tumors, and neuroblastoma } \\ \text { NCT02097810 } & \text { Entrectinib } & \text { I } & \text { Locally advanced solid tumors, metastatic solid tumors }\end{array}$

NCT02587650 Entrectinib

II

NCT02576431 Larotrectinib

NCT02637687 Larotrectinib NCT03213704 Larotrectinib

II
stage IV cutaneous melanoma AJCC v6 and v7 melanomas $\begin{array}{cl}\text { I and II } & \text { Neoplasms, central nervous system neoplasms } \\ \text { II } & \text { Advanced malignant solid neoplasm, malignant glioma, ann arbor stage }\end{array}$
ALK fusion protein expression, BRAF wt Allele, invasive skin melanoma, MET fusion gene positive, NRAS wt Allele, NTRK1 fusion positive, NTRK2 fusion positive, NTRK3 fusion positive, RET fusion positive, ROS1 fusion positive, stage III cutaneous melanoma AJCC v7, stage IIIA cutaneous melanoma AJCC v7, stage IIIB cutaneous melanoma AJCC v7, stage IIIC cutaneous melanoma AJCC v7, and

II Non-small-cell lung carcinoma, thyroid neoplasms, sarcoma, colorectal neoplasms, salivary gland neoplasms, biliary tract neoplasms, brain neoplasm, primary, carcinoma, ductal, breast, melanoma, solid tumors, glioblastoma, bile duct neoplasms, astrocytoma, head and neck squamous cell carcinoma, pontine glioma, pancreatic neoplasms, ovarian neoplasms, renal cell carcinoma, cholangiocarcinoma, bronchogenic carcinoma, bronchial neoplasms, lung neoplasms, respiratory tract neoplasms, thoracic neoplasms, neoplasms, nerve tissue, nevi and III childhood non-hodgkin lymphoma, ann arbor stage IV childhood non-hodgkin lymphoma, malignant glioma, NTRK1 fusion positive, NTRK2 fusion positive, NTRK3 fusion positive, recurrent central nervous system neoplasm, recurrent childhood ependymoma, recurrent childhood malignant germ cell tumor, recurrent childhood medulloblastoma, recurrent childhood non-hodgkin lymphoma, recurrent childhood rhabdomyosarcoma, recurrent childhood soft tissue sarcoma, recurrent ewing sarcoma, recurrent glioma, recurrent hepatoblastoma, recurrent langerhans cell histiocytosis, recurrent malignant solid neoplasm, recurrent neuroblastoma, recurrent osteosarcoma, recurrent peripheral primitive neuroectodermal tumor, refractory central nervous system neoplasm, refractory childhood malignant germ cell tumor, refractory langerhans cell histiocytosis, refractory malignant solid neoplasm, refractory neuroblastoma, refractory non-hodgkin lymphoma, rhabdoid tumor, stage III osteosarcoma AJCC v7, stage III soft tissue sarcoma AJCC v7, stage IV osteosarcoma AJCC v7, stage IV soft tissue sarcoma AJCC v7, stage IVA osteosarcoma AJCC v7, stage IVB osteosarcoma AJCC v7, and wilms tumor

NCT02122913 Larotrectinib I Unspecified adult solid tumor, protocol specific
Recruiting

Recruiting

Completed

Recruiting

Active, not recruiting

Recruiting

Recruiting

NCT, National Clinical Trials.

maturation of peripheral T-cell (102). As a result, when we target BDNF/TrkB for cancer treatment, a close monitoring of T-cell activity is necessary so that the antitumor immune response won't be attenuated. 


\section{Conclusion and discussion}

BDNF plays an important role in a wide range of cancers by binding its receptor TrkB (6-12). Up-regulation of $\mathrm{BDNF} / \mathrm{TrkB}$ results in a series of downstream signalings, including PI3K/Akt, Ras-Raf-MEK-ERK, PLC $\gamma$ pathway, and transactivation of EGFR, etc (Fig. 2). Stimulation of these signalings exerts oncogenic effects by mediating cancer cell's growth, proliferation, survival, migration, epithelial to mesenchymal trasition, anoikis, relapse and chemotherapeutic sensitivity (6-12). Although BDNF/TrkB regulates several downstream signalings and these pathways may correspond to each other, not all these signalings will be stimulated in response to BDNF/TrkB. For example, it was reported that BDNF can rescue neuroblastoma cells from etoposide. Inhibition of PI3K but not MAPK can abrogate this ability, indicating MAPK pathway may not be involved in this oncogenic role (103).

Entrectinib (90), Larotrectinib (91), and Cabozaninib (93) are three drugs targeting Trk receptors for treatment of cancer and the clinical trial results are promising. In some cancers, patients have increased objective response rate, median progression-free survival rate and overall survival rate. Considering the three drugs can all penetrate BBB, we should pay close attention to their side effects on central nervous system. Larotrectinib has shown toxicity profile with fatigue, dizziness and memory loss (92). Besides, BDNF in central nervous system, especially hypothalamus, have an oncolytic role by increasing T-cell toxicity $(98,100,101)$. When targeting Trk receptors for treatment of cancer, these drugs will also decrease activity of BDNF/TrkB in the central nervous system and thus may attenuate BDNF-mediated anti-cancer immune response. A close monitoring of T-cell activity is therefore necessary.

Since BDNF plays a crucial role in oncogenesis, is it still safe to activate BDNF/TrkB for treatment of some neurodegenerative diseases? Like human hormones, too much or too little of BDNF may be harmful. It is not suggested that healthy people should take BDNF as a supplement due to the potential risk of oncogenesis. Nevertheless, administration of BDNF may be beneficial in some aging and neurodegenerative diseases, such as amyotrophic lateral sclerosis (ALS), peripheral neuropathy, Parkinson's disease and Alzheimer's disease (104-106). Under these unhealthy conditions, $\mathrm{BNDF} / \mathrm{TrkB}$ is down-regulated and there is no evidence that administration of BDNF can cause cancer. For clinical application, BDNF is not directly administrated since it is a moderately-sized and charged protein, and can't easily cross BBB. Therefore, scientists have spent decades trying to establish small drugs that could penetrate BBB and safely augment BDNF levels in the brain. Recently, Ampakines, a modulator of $\alpha$-amino-3-hydroxy-5-methyl-4-isoxazolepropi onic acid (AMPA) receptor, have been found to significantly elevate BDNF levels in some brain regions and also successfully correct age-related memory issues without severe side effects $(96,107)$.

\section{Acknowledgements}

Not applicable.

\section{Funding}

The present review was supported by the National Science Foundation of China (grant no. 81570344).

\section{Availability of data and materials}

Not applicable.

\section{Authors' contributions}

LM, BL and RJ wrote the manuscript. XJ and XY critically revised the manuscript for important intellectual content. YX reviewed and edited the manuscript.

\section{Ethics approval and consent to participate}

Not applicable.

\section{Patient consent for publication}

Not applicable.

\section{Competing interests}

The authors declare that they have no competing interests.

\section{References}

1. Bibel M and Barde YA: Neurotrophins: Key regulators of cell fate and cell shape in the vertebrate nervous system. Genes Dev 14: 2919-2937, 2000.

2. Huang EJ and Reichardt LF: Neurotrophins: Roles in neuronal development and function. Annu Rev Neurosci 24: 677-736, 2001.

3. Meldolesi J: Neurotrophin receptors in the pathogenesis, diagnosis and therapy of neurodegenerative diseases. Pharmacol Res 121: 129-137, 2017.

4. Reichardt LF: Neurotrophin-regulated signalling pathways. Rev Physiol Biochem Pharmacol 361: 1545-1564, 2006.

5. Meldolesi J: Neurotrophin Trk receptors: New targets for cancer therapy. Rev Physiol Biochem Pharmacol 174: 67-79, 2018.

6. Vanhecke E, Adriaenssens E, Verbeke S, Meignan S, Germain E, Berteaux N, Nurcombe V, Le Bourhis X and Hondermarck H: Brain-derived neurotrophic factor and neurotrophin-4/5 are expressed in breast cancer and can be targeted to inhibit tumor cell survival. Clin Cancer Res 17: 1741-1752, 2011.

7. Ricci A, Graziano P, Mariotta S, Cardillo G, Sposato B, Terzano C and Bronzetti E: Neurotrophin system expression in human pulmonary carcinoid tumors. Growth Factors 23: 303-312, 2005.

8. Moon A, Won KY, Lee JY, Kang I, Lee SK and Lee J: Expression of BDNF, TrkB, and p53 in early-stage squamous cell carcinoma of the uterine cervix. Pathology 43: 453-458, 2011.

9. Brunetto de Farias C, Rosemberg DB, Heinen TE, Koehler-Santos P, Abujamra AL, Kapczinski F, Brunetto AL, Ashton-Prolla P, Meurer L, Reis Bogo M, et al: BDNF/TrkB content and interaction with gastrin-releasing peptide receptor blockade in colorectal cancer. Oncology 79: 430-439, 2010.

10. Gilbertson RJ and Rich JN: Making a tumour's bed: Glioblastoma stem cells and the vascular niche. Nat Rev Cancer 7: 733-736, 2007.

11. Tanaka K, Okugawa Y, Toiyama Y, Inoue Y, Saigusa S, Kawamura M, Araki T, Uchida K, Mohri Y and Kusunoki M: Brain-derived neurotrophic factor (BDNF)-induced tropomyosin-related kinase B (Trk B) signaling is a potential therapeutic target for peritoneal carcinomatosis arising from colorectal cancer. PLoS One 9: e96410, 2014.

12. Okamura K, Harada T, Wang S, Ijichi K, Furuyama K, Koga T, Okamoto T, Takayama K, Yano T and Nakanishi Y: Expression of TrkB and BDNF is associated with poor prognosis in non-small cell lung cancer. Lung Cancer 78: 100-106, 2012. 
13. Lange $A M$ and Lo HW: Inhibiting TRK proteins in clinical cancer therapy. Cancers (Basel) 10: pii: E105, 2018.

14. Tanaka J, Horiike Y, Matsuzaki M, Miyazaki T, Ellis-Davies GC and Kasai H: Protein synthesis and neurotrophin-dependent structural plasticity of single dendritic spines. Science 319: 1683-1687, 2008.

15. Horch HW and Katz LC: BDNF release from single cells elicits local dendritic growth in nearby neurons. Nat Neurosci 5: $1177-1184,2002$

16. Figurov A, Pozzo-Miller LD, Olafsson P, Wang T and Lu B: Regulation of synaptic responses to high-frequency stimulation and LTP by neurotrophins in the hippocampus. Nature 381: 706-709, 1996.

17. Xu B, Goulding EH, Zang K, Cepoi D, Cone RD, Jones KR, Tecott LH and Reichardt LF: Brain-derived neurotrophic factor regulates energy balance downstream of melanocortin-4 receptor. Nat Neurosci 6: 736-742, 2003.

18. Cao L, Lin EJ, Cahill MC, Wang C, Liu X and During MJ: Molecular therapy of obesity and diabetes by a physiological autoregulatory approach. Nat Med 15: 447-454, 2009.

19. Meng L, Huang T, Sun C, Hill DL and Krimm R: BDNF is required for taste axon regeneration following unilateral chorda tympani nerve section. Exp Neurol 293: 27-42, 2017.

20. Meng L, Ohman-Gault L, Ma L and Krimm RF: Taste bud-derived BDNF is required to maintain normal amounts of innervation to adult taste buds. eNeuro 2: pii: ENEURO.0097-15.2015, 2015.

21. Meng L, Jiang X and Ji R: Role of neurotrophin in the taste system following gustatory nerve injury. Metab Brain Dis 30: 605-613,2015.

22. Ji R, Meng L, Li Q and Lu Q: TAM receptor deficiency affects adult hippocampal neurogenesis. Metab Brain Dis 30: 633-644, 2015.

23. Ji R, Meng L, Jiang X, Cvm NK, Ding J, Li Q and Lu Q: TAM receptors support neural stem cell survival, proliferation and neuronal differentiation. PLoS One 9: e115140, 2014.

24. Li Q, Ford MC, Lavik EB and Madri JA: Modeling the neurovascular niche: VEGF- and BDNF-mediated cross-talk between neural stem cells and endothelial cells: An in vitro study. J Neurosci Res 84: 1656-1668, 2006.

25. Blurton-Jones M, Kitazawa M, Martinez-Coria H, Castello NA Müller FJ, Loring JF, Yamasaki TR, Poon WW, Green KN and LaFerla FM: Neural stem cells improve cognition via BDNF in a transgenic model of Alzheimer disease. Proc Natl Acad Sci USA 106: 13594-13599, 2009.

26. Pearse RN, Swendeman SL, Li Y, Rafii D and Hempstead BL: A neurotrophin axis in myeloma: TrkB and BDNF promote tumor-cell survival. Blood 105: 4429-4436, 2005.

27. Nakagawara A: Trk receptor tyrosine kinases: A bridge between cancer and neural development. Cancer Lett 169 107-114, 2001

28. Brodeur GM, Minturn JE, Ho R, Simpson AM, Iyer R, Varela CR Light JE, Kolla V and Evans AE: Trk receptor expression and inhibition in neuroblastomas. Clin Cancer Res 15: 3244-3250, 2009.

29. Porter AC and Vaillancourt RR: Tyrosine kinase receptor-activated signal transduction pathways which lead to oncogenesis. Oncogene 17 (11 Reviews): 1343-1352, 1998.

30. Nakagawara A, Azar CG, Scavarda NJ and Brodeur GM: Expression and function of TRK-B and BDNF in human neuroblastomas. Mol Cell Biol 14: 759-767, 1994.

31. Yin B, Ma ZY, Zhou ZW, Gao WC, Du ZG, Zhao ZH and Li QQ: The TrkB+ cancer stem cells contribute to post-chemotherapy recurrence of triple-negative breast cancers in an orthotopic mouse model. Oncogene 34: 761-770, 2015

32. Zhang SY, Hui LP, Li CY, Gao J, Cui ZS and Qiu XS: More expression of BDNF associates with lung squamous cell carcinoma and is critical to the proliferation and invasion of lung cancer cells. BMC Cancer 16: 171, 2016.

33. Radin DP and Patel P: BDNF: An oncogene or tumor suppressor? Anticancer Res 37: 3983-3990, 2017.

34. Hwang JJ, Park MH, Choi SY and Koh JY: Activation of the Trk signaling pathway by extracellular zinc. Role of metalloproteinases. J Biol Chem 280: 11995-12001, 2005.

35. Hempstead BL: Dissecting the diverse actions of pro-and mature neurotrophins. Curr Alzheimer Res 3: 19-24, 2006.

36. Johnston AL, Lun X, Rahn JJ, Liacini A, Wang L, Hamilton MG, Parney IF, Hempstead BL, Robbins SM, Forsyth PA, et al: The p75 neurotrophin receptor is a central regulator of glioma invasion. PLoS Biol 5: e212, 2007.
37. Herrmann JL, Menter DG, Hamada J, Marchetti D, Nakajima M and Nicolson GL: Mediation of NGF-stimulated extracellular matrix invasion by the human melanoma low-affinity p75 neurotrophin receptor: melanoma $\mathrm{p} 75$ functions independently of trkA. Mol Biol Cell 4: 1205-1216, 1993.

38. Marchetti D, Aucoin R, Blust J, Murry B and Greiter-Wilke A: p75 neurotrophin receptor functions as a survival receptor in brain-metastatic melanoma cells. J Cell Biochem 91: 206-215, 2004.

39. Khwaja F and Djakiew D: Inhibition of cell-cycle effectors of proliferation in bladder tumor epithelial cells by the p75NTR tumor suppressor. Mol Carcinog 36: 153-160, 2003.

40. Yuanlong H, Haifeng J, Xiaoyin Z, Jialin S, Jie L, Li Y, Huahong X, Jiugang S, Yanglin P, Kaichun W, et al: The inhibitory effect of p75 neurotrophin receptor on growth of human hepatocellular carcinoma cells. Cancer Lett 268: 110-119, 2008.

41. Jin H, Pan Y, He L, Zhai H, Li X, Zhao L, Sun L, Liu J, Hong L, Song J, et al: p75 neurotrophin receptor inhibits invasion and metastasis of gastric cancer. Mol Cancer Res 5: 423-433, 2007.

42. Ji R, Tian S, Lu HJ, Lu Q, Zheng Y, Wang X, Ding J, Li Q and Lu Q: TAM receptors affect adult brain neurogenesis by negative regulation of microglial cell activation. J Immunol 191: 6165-6177, 2013

43. Puehringer D, Orel N, Lüningschrör P, Subramanian N, Herrmann T, Chao MV and Sendtner M: EGF transactivation of Trk receptors regulates the migration of newborn cortical neurons. Nat Neurosci 16: 407-415, 2013.

44. Park JR, Eggert A and Caron H: Neuroblastoma: Biology, prognosis, and treatment. Hematol Oncol Clin North Am 24: 65-86, 2010.

45. Kermani P and Hempstead B: Brain-derived neurotrophic factor: A newly described mediator of angiogenesis. Trends Cardiovasc Med 17: 140-143, 2007.

46. Kermani P, Rafii D, Jin DK, Whitlock P, Schaffer W, Chiang A, Vincent L, Friedrich M, Shido K, Hackett NR, et al: Neurotrophins promote revascularization by local recruitment of TrkB+ endothelial cells and systemic mobilization of hematopoietic progenitors. J Clin Invest 115: 653-663, 2005.

47. Ho R, Eggert A, Hishiki T, Minturn JE, Ikegaki N, Foster P, Camoratto AM, Evans AE and Brodeur GM: Resistance to chemotherapy mediated by TrkB in neuroblastomas. Cancer Res 62: 6462-6466, 2002.

48. Brierley GV, Priebe IK, Purins L, Fung KY, Tabor B, Lockett T, Nice E, Gibbs P, Tie J, McMurrick P, et al: Serum concentrations of brain-derived neurotrophic factor (BDNF) are decreased in colorectal cancer patients. Cancer Biomark 13: 67-73, 2013.

49. Xiong L, Deng X, Wen Y, Yang Z and Miao X: Association of BDNF and BMPR1A with clinicopathologic parameters in benign and malignant gallbladder lesions. World J Surg Oncol 11: 80, 2013.

50. Choi B, Lee EJ, Shin MK, Park YS, Ryu MH, Kim SM, Kim EY, Lee HK and Chang EJ: Upregulation of brain-derived neurotrophic factor in advanced gastric cancer contributes to bone metastatic osteolysis by inducing long pentraxin 3 . Oncotarget 7: 55506-55517, 2016

51. Götz R and Sendtner M: Cooperation of tyrosine kinase receptor TrkB and epidermal growth factor receptor signaling enhances migration and dispersal of lung tumor cells. PLoS One 9: e100944, 2014

52. Qiu L, Zhou C, Sun Y, Di W, Scheffler E, Healey S, Kouttab N, Chu W and Wan Y: Crosstalk between EGFR and TrkB enhances ovarian cancer cell migration and proliferation. Int J Oncol 29: 1003-1011, 2006.

53. de Farias CB, Heinen TE, dos Santos RP, Abujamra AL, Schwartsmann $\mathrm{G}$ and Roesler R: BDNF/TrkB signaling protects HT-29 human colon cancer cells from EGFR inhibition. Biochem Biophys Res Commun 425: 328-332, 2012.

54. Heinen TE, dos Santos RP, da Rocha A, Dos Santos MP, Lopez PL, Silva Filho MA, Souza BK, Rivero LF, Becker RG, Gregianin LJ, et al: Trk inhibition reduces cell proliferation and potentiates the effects of chemotherapeutic agents in Ewing sarcoma. Oncotarget 7: 34860, 2016.

55. Yee CL, Jones KR and Finger TE: Brain-derived neurotrophic factor is present in adult mouse taste cells with synapses. J Comp Neurol 459: 15-24, 2003.

56. Makino K, Kawamura K, Sato W, Kawamura N, Fujimoto T and Terada Y: Inhibition of uterine sarcoma cell growth through suppression of endogenous tyrosine kinase B signaling. PLoS One 7: e41049, 2012 
57. Iyer R, Evans AE, Qi X, Ho R, Minturn JE, Zhao H, Balamuth N, Maris JM and Brodeur GM: Lestaurtinib enhances the antitumor efficacy of chemotherapy in murine xenograft models of neuroblastoma. Clin Cancer Res 16: 1478-1485, 2010.

58. Iyer R, Wehrmann L, Golden RL, Naraparaju K, Croucher JL, MacFarland SP, Guan P, Kolla V, Wei G, Cam N, et al: Entrectinib is a potent inhibitor of Trk-driven neuroblastomas in a xenograft mouse model. Cancer Lett 372: 179-186, 2016.

59. Jaboin J, Kim CJ, Kaplan DR and Thiele CJ: Brain-derived neurotrophic factor activation of TrkB protects neuroblastoma cells from chemotherapy-induced apoptosis via phosphatidylinositol 3'-kinase pathway. Cancer Res 62: 6756-6763, 2002.

60. Bao W, Qiu H, Yang T, Luo X, Zhang H and Wan X: Upregulation of TrkB promotes epithelial-mesenchymal transition and anoikis resistance in endometrial carcinoma. PLoS One 8: e70616, 2013.

61. Kupferman M, Jiffar T, El-Naggar A, Yilmaz T, Zhou G, Xie T, Feng L, Wang J, Holsinger FC, Yu D and Myers JN: TrkB induces EMT and has a key role in invasion of head and neck squamous cell carcinoma. Oncogene 29: 2047-2059, 2010.

62. Ricci A, De Vitis C, Noto A, Fattore L, Mariotta S, Cherubini E, Roscilli G, Liguori G, Scognamiglio G, Rocco G, et al: TrkB is responsible for EMT transition in malignant pleural effusions derived cultures from adenocarcinoma of the lung. Cell Cycle 12: 1696-1703, 2013.

63. Smit MA, Geiger TR, Song JY, Gitelman I and Peeper DS: A Twist-Snail axis critical for TrkB-induced epithelial-mesenchymal transition-like transformation, anoikis resistance, and metastasis. Mol Cell Biol 29: 3722-3737, 2009.

64. Lee J, Jiffar T and Kupferman ME: A novel role for BDNF-TrkB in the regulation of chemotherapy resistance in head and neck squamous cell carcinoma. PLoS One 7: e30246, 2012

65. Xia H, Li Y and Lv X: MicroRNA-107 inhibits tumor growth and metastasis by targeting the BDNF-mediated PI3K/AKT pathway in human non-small lung cancer. Int J Oncol 49 : 1325-1333, 2016.

66. DeWitt J, Ochoa V, Urschitz J, Elston M, Moisyadi S and Nishi R: Constitutively active TrkB confers an aggressive transformed phenotype to a neural crest-derived cell line. Oncogene 33 977-985, 2014

67. Chen B, Liang Y, He Z, An Y, Zhao W and Wu J: Autocrine activity of BDNF induced by the STAT3 signaling pathway causes prolonged TrkB activation and promotes human non-small-cell lung cancer proliferation. Sci Rep 6: 30404, 2016.

68. Navé BT, Ouwens M, Withers DJ, Alessi DR and Shepherd PR Mammalian target of rapamycin is a direct target for protein kinase B: Identification of a convergence point for opposing effects of insulin and amino-acid deficiency on protein translation. Biochem J 344: 427-431, 1999.

69. Aoki M, Blazek E and Vogt PK: A role of the kinase mTOR in cellular transformation induced by the oncoproteins P3k and Akt. Proc Natl Acad Sci USA 98: 136-141, 2001.

70. Martens LK, Kirschner KM, Warnecke C and Scholz H: Hypoxia-inducible factor-1 (HIF-1) is a transcriptional activator of the TrkB neurotrophin receptor gene. J Biol Chem 282: $14379-14388,2007$.

71. Li Z, Zhang J, Liu Z, Woo CW and Thiele CJ: Downregulation of Bim by brain-derived neurotrophic factor activation of TrkB protects neuroblastoma cells from paclitaxel but not etoposide or cisplatin-induced cell death. Cell Death Differ 14: 318-326, 2007.

72. Radin D, Lippa A, Patel P and Leonardi D: Lifeguard inhibition of Fas-mediated apoptosis: A possible mechanism for explaining the cisplatin resistance of triple-negative breast cancer cells Biomed Pharmacother 77: 161-166, 2016.

73. Voldborg BR, Damstrup L, Spang-Thomsen M and Poulsen HS Epidermal growth factor receptor (EGFR) and EGFR mutations, function and possible role in clinical trials. Ann Oncol 8: 1197-1206, 1997

74. Hunter T and Pines J: Cyclins and cancer. II: Cyclin D and CDK inhibitors come of age. Cell 79: 573-582, 1994

75. Bos JL: Ras oncogenes in human cancer: A review. Cancer Res 49: 4682-4689, 1989.

76. Davis RJ: The mitogen-activated protein kinase signal transduction pathway. J Biol Chem 268: 14553-14556, 1993.

77. Sinkevicius KW, Kriegel C, Bellaria KJ, Lee J, Lau AN, Leeman KT, Zhou P, Beede AM, Fillmore CM, Caswell D, et al: Neurotrophin receptor TrkB promotes lung adenocarcinoma metastasis. Proc Natl Acad Sci USA 111: 10299-10304, 2014.
78. Davis NM, Sokolosky M, Stadelman K, Abrams SL, Libra M, Candido S, Nicoletti F, Polesel J, Maestro R, D'Assoro A, et al: Deregulation of the EGFR/PI3K/PTEN/Akt/mTORC1 pathway in breast cancer: Possibilities for therapeutic intervention. Oncotarget 5: 4603-4650, 2014.

79. Morris LG, Taylor BS, Bivona TG, Gong Y, Eng S, Brennan CW, Kaufman A, Kastenhuber ER, Banuchi VE, Singh B, et al: Genomic dissection of the epidermal growth factor receptor (EGFR)/PI3K pathway reveals frequent deletion of the EGFR phosphatase PTPRS in head and neck cancers. Proc Natl Acad Sci USA 108: 19024-19029, 2011.

80. Lee DH, Szczepanski MJ and Lee YJ: Magnolol induces apoptosis via inhibiting the EGFR/PI3K/Akt signaling pathway in human prostate cancer cells. J Cell Biochem 106: 1113-1122, 2009.

81. Cowley S, Paterson H, Kemp P and Marshall CJ: Activation of MAP kinase kinase is necessary and sufficient for PC12 differentiation and for transformation of NIH 3T3 cells. Cell 77: 841-852, 1994.

82. Huang EJ and Reichardt LF: Trk receptors: Roles in neuronal signal transduction. Annu Rev Biochem 72: 609-642, 2003.

83. Siu MK, Wong OG and Cheung AN: TrkB as a therapeutic target for ovarian cancer. Expert Opin Ther Targets 13: 1169-1178, 2009.

84. Yuan Y, Ye HQ and Ren QC: Upregulation of the BDNF/TrKB pathway promotes epithelial-mesenchymal transition, as well as the migration and invasion of cervical cancer. Int J Oncol 52: 461-472, 2018.

85. Cazorla M, Jouvenceau A, Rose C, Guilloux JP, Pilon C, Dranovsky A and Prémont J: Cyclotraxin-B, the first highly potent and selective TrkB inhibitor, has anxiolytic properties in mice. PLoS One 5: e9777, 2010.

86. Cazorla M, Prémont J, Mann A, Girard N, Kellendonk C and Rognan D: Identification of a low-molecular weight TrkB antagonist with anxiolytic and antidepressant activity in mice. J Clin Invest 121: 1846-1857, 2011

87. Light JE, Koyama H, Minturn JE, Ho R, Simpson AM, Iyer R, Mangino JL, Kolla V, London WB and Brodeur GM: Clinical significance of NTRK family gene expression in neuroblastomas. Pediatr Blood Cancer 59: 226-232, 2012.

88. Bertrand T, Kothe M, Liu J, Dupuy A, Rak A, Berne PF, Davis S, Gladysheva T, Valtre C, Crenne JY and Mathieu M: The crystal structures of TrkA and TrkB suggest key regions for achieving selective inhibition. J Mol Biol 423: 439-453, 2012.

89. Drilon A, Siena S, Ou SI, Patel M, Ahn MJ, Lee J, Bauer TM, Farago AF, Wheler JJ, Liu SV, et al: Safety and antitumor activity of the multitargeted pan-TRK, ROS1, and ALK inhibitor entrectinib: Combined results from two phase I trials (ALKA-372-001 and STARTRK-1). Cancer Discov 7: 400-409, 2017.

90. Ardini E, Menichincheri M, Banfi P, Bosotti R, De Ponti C, Pulci R, Ballinari D, Ciomei M, Texido G, Degrassi A, et al: Entrectinib, a pan-TRK, ROS1, and ALK inhibitor with activity in multiple molecularly defined cancer indications. Mol Cancer Ther 15: 628-639, 2016 .

91. Burris HA, Shaw AT, Bauer TM, Farago AF, Doebele RC Smith S, Nanda N, Cruickshank S, Low JA and Brose MS: Pharmacokinetics (PK) of LOXO-101 during the first-in-human phase I study in patients with advanced solid tumors: Interim update. Cancer Res 75: 4529, 2015.

92. Drilon A, Laetsch TW, Kummar S, DuBois SG, Lassen UN, Demetri GD, Nathenson M, Doebele RC, Farago AF, Pappo AS, et al: Efficacy of larotrectinib in TRK fusion-positive cancers in adults and children. N Engl J Med 378: 731-739, 2018.

93. Al-Salama ZT and Keating GM: Cabozantinib: A review in advanced renal cell carcinoma. Drugs 76: 1771-1778, 2016.

94. Abdelaziz A and Vaishampayan U: Cabozantinib for renal cell carcinoma: Current and future paradigms. Curr Treat Options Oncol 18: 18, 2017.

95. Schiff D, Desjardins A, Cloughesy T, Mikkelsen T, Glantz M, Chamberlain MC, Reardon DA and Wen PY: Phase 1 dose escalation trial of the safety and pharmacokinetics of cabozantinib concurrent with temozolomide and radiotherapy or temozolomide after radiotherapy in newly diagnosed patients with high-grade gliomas. Cancer 122: 582-587, 2016.

96. Radin DP, Zhong S, Purcell R and Lippa A: Acute ampakine treatment ameliorates age-related deficits in long-term potentiation. Biomed Pharmacother 84: 806-809, 2016.

97. Morgensztern D, Campo MJ, Dahlberg SE, Doebele RC, Garon E, Gerber DE, Goldberg SB, Hammerman PS, Heist RS, Hensing T, et al: Molecularly targeted therapies in non-small-cell lung cancer annual update 2014. J Thorac Oncol 10 (1 Suppl 1): S1-S63, 2015 
98. Cao L, Liu X, Lin EJ, Wang C, Choi EY, Riban V, Lin B and During MJ: Environmental and genetic activation of a brain-adipocyte BDNF/leptin axis causes cancer remission and inhibition. Cell 142: 52-64, 2010.

99. Liu X, McMurphy T, Xiao R, Slater A, Huang W and Cao L: Hypothalamic gene transfer of BDNF inhibits breast cancer progression and metastasis in middle age obese mice. Mol Ther 22: 1275-1284, 2014

100. Xiao R, Bergin SM, Huang W, Slater AM, Liu X, Judd RT, Lin ED, Widstrom KJ, Scoville SD, Yu J, et al: Environmental and genetic activation of hypothalamic BDNF modulates T-cell immunity to exert an anticancer phenotype. Cancer Immunol Res 4: 488-497, 2016.

101. Garofalo S, D'Alessandro G, Chece G, Brau F, Maggi L, Rosa A, Porzia A, Mainiero F, Esposito V, Lauro C, et al: Enriched environment reduces glioma growth through immune and non-immune mechanisms in mice. Nat Commun 6: 6623, 2015.

102. Linker RA, Lee DH, Flach AC, Litke T, van den Brandt J, Reichardt HM, Lingner T, Bommhardt U, Sendtner M, Gold R, et al: Thymocyte-derived BDNF influences T-cell maturation at the DN3/DN4 transition stage. Eur J Immunol 45: 1326-1338, 2015.
103. Jaboin J, Hong A, Kim CJ and Thiele CJ: Cisplatin-induced cytotoxicity is blocked by brain-derived neurotrophic factor activation of TrkB signal transduction path in neuroblastoma. Cancer Lett 193: 109-114, 2003.

104. Ochs G, Penn RD, York M, Giess R, Beck M, Tonn J, Haigh J, Malta E, Traub M, Sendtner M and Toyka KV: A phase I/II trial of recombinant methionyl human brain derived neurotrophic factor administered by intrathecal infusion to patients with amyotrophic lateral sclerosis. Amyotroph Lateral Scler Other Motor Neuron Disord 1: 201-206, 2000.

105. Apfel SC, Kessler JA, Adornato BT, Litchy WJ, Sanders C and Rask CA: Recombinant human nerve growth factor in the treatment of diabetic polyneuropathy. NGF Study Group. Neurology 51: 695-702, 1998.

106. Kordower JH, Palfi S, Chen EY, Ma SY, Sendera T, Cochran EJ, Cochran EJ, Mufson EJ, Penn R, Goetz CG and Comella CD: Clinicopathological findings following intraventricular glial-derived neurotrophic factor treatment in a patient with Parkinson's disease. Ann Neurol 46: 419-424, 1999.

107. Radin DP, Purcell R and Lippa AS: Oncolytic properties of ampakines in vitro. Anticancer Res 38: 265-269, 2018. 